\title{
DOS ESPECIES NUEVAS DEL GÉNERO ACHROMOPORUS (DIPLOPODA: POLYDESMIDA: CHELODESMIDAE) DEL PARQUE NACIONAL JOSÉ DEL CARMEN RAMÍREZ
}

\author{
Carlos Suriel \\ Museo Nacional de Historia Natural, Santo Domingo. Calle César Nicolás Penson, Plaza de la Cultura, \\ Santo Domingo, República Dominicana. c.suriel@museohistorianatural.gov.do
}

\section{RESUMEN}

Se describen dos especies nuevas del género Achromoporus Loomis, 1936, colectadas en el Parque Nacional José del Carmen Ramírez, en la Cordillera Central de la República Dominicana. Con estas se eleva a 24 el número de especies del género Achromoporus para La Hispaniola, asumiendo las últimas sinonimias sugeridas por Pérez-Asso (2009).

Palabras clave: Achromoporus, Chelodesmidae, Diplopoda, especies nuevas, Parque Nacional José del Carmen Ramírez, República Dominicana, La Hispaniola.

\section{ABSTRACT}

Two new milliped species of the genus Achromoporus Loomis, 1936, are described from Parque Nacional José del Carmen Ramírez, Cordillera Central, Dominican Republic. The number of Achromoporus species from Hispaniola is increased to 24, according to last synonymous proposed by Pérez-Asso (2009).

Key words: Achromoporus, Chelodesmidae, Diplopoda, new species, Parque Nacional José del Carmen Ramírez, Dominican Republic, Hispaniola.

\section{INTRODUCCIÓN}

El género Achromoporus fue descrito por Loomis (1936) con la especie tipo A. coloratus, junto a esta también describe $A$. enneryensis, A. robustus y $A$. furcipes, todas de Haití; posteriormente, el mismo autor (1941) describe $A$. heteromus para la República Dominicana. PérezAsso (2005) describe A. ebanoverde, A. bayaguana y A. coloratus neiba para la República Dominicana. El rasgo distintivo fundamental del género Achromoporus ha sido siempre el acropodito o fémur bifurcado, sin embargo, Loomis (1941, Ídem) describe otros cuatro nuevos géneros de la familia Chelodesmidae con el acropodito birramoso, pero usando también otras características como definitorias: Lasiomazus, Craterodesmus, Biaporus y Synecheporus. La no consideración del rasgo determinante de un género previo en la descripción posterior de géneros nuevos originó confusión, la cual se ha hecho mayor, como era previsible, en la medida que han aparecido nuevas especies con gradaciones y combinaciones de las características utilizadas. Un trabajo reciente sobre el género (Pérez-Asso, 2009), trata de resolver los problemas proponiendo tomar la condición de acropodito birramoso como rasgo definitorio y unificador de Achromoporus, sugiriendo como nuevos sinónimos, en base al criterio de antigüedad, los posteriores géneros descritos por Loomis con esta característica, es decir, Lasiomazus, Craterodesmus, Biaporus y Synecheporus; al mismo tiempo, este autor describe 11 especies nuevas del género.

Con las sinonimias sugeridas para las especies tipo de los cuatro géneros referidos, junto a las 11 especies nuevas descritas, la invalidación de la subespecie $A$. coloratus neiba, la que pasa como sinónimo de $A$. coloratus Loomis (Pérez-Asso, 2009, Ídem), así como la descripción previa de $A$. ebanoverde y A. bayaguana (Pérez-Asso, 2005, Ídem) se reúne un total de 22 especies. De manera que con las dos descritas en el presente artículo totalizan 24 las especies del género Achromoporus, resultando en el taxon más diverso dentro de la familia Chelodesmidae en La Hispaniola y uno de los táxones más interesantes para estudios futuros. 


\title{
MATERIALES Y MÉTODOS
}

Las observaciones se realizaron con un microscopio estereoscópico MEIJI TECHNO modelo EMZ-5TR. Los especímenes utilizados se colectaron en pinares y manchas de bosques latifoliados del Parque Nacional José del Carmen Ramírez durante expediciones realizadas en fechas (26-29)-vi-08 y (21-25)-xi-09; fueron preservados en alcohol etílico al 70\%. Para dibujar los gonopodos completos y el dorso del cuerpo el autor se valió de fotos tomadas con una cámara Nikon, modelo Coolpix 4500 adaptada a un microscopio estereoscópico marca Leica, modelo MZ7.5, con acercamientos entre $0.63 \mathrm{x}$ y $5.0 \mathrm{x}$, lentes objetivos de $0.5 \mathrm{x}$ y $1.0 \mathrm{x}$, procesamiento de las imágenes con el programa Auto Montage versión 5.0 y edición con PhotoShop versión 7.0. Los dibujos de las piezas gonopodales separadas se hicieron con una cámara lúcida marca LEITZ WETZLAR. En las ilustraciones de los gonopodos el autor sombreó el proceso prefemoral a fin de destacarlo, difereciándolo del acropodito. Todos los dibujos se hicieron manualmente. Para las mediciones se usaron una Miniescala de Bio Quip Products, Inc. modelo "Métrica" con un rango de $5 \mathrm{~mm}$ y divisiones de $0.1 \mathrm{~mm}$ y una regla milimetrada transparente. Para la categorización y nomenclatura taxonómica se remite a Suriel (2009).

La longitud del cuerpo se tomó desde el extremo de la cabeza hasta el ápice del epiprocto y el ancho entre los ángulos posteriores de los paraterguitos en el segmento 7 (Pérez-Asso, 1996). Se ofrece la media de las medidas obtenidas, indicándose entre paréntesis rango de variación y número de ejemplares. El ancho de la cabeza se tomó siguiendo la línea imaginaria que une las genas. Las coordenadas geográficas y la altitud de las localidades se registraron usando un Global Position System (GPS) marca Garmin, modelo ETREX. Abreviaturas: MNHNSD (Museo Nacional de Historia Natural, Santo Domingo), SX ( $\mathrm{S}=$ diplosegmento, $\mathrm{X}=$ número del diplosegmento correspondiente), APP (ángulo posterior del paraterguito) y AAP (ángulo anterior del paraterguito).

\section{RESULTADOS}

\author{
CLASIFICACIÓN
}

Familia Chelodesmidae Cook, 1895

Subfamilia Chelodesminae Hoffman, 1979

Género Achromoporus Loomis, 1936 (sensu Pérez-Asso, 2009)

Referencias consideradas. Achromoporus Loomis, 1936, Bull. Mus. Comp. Zool., 80 (1):112-119. Jeekel, 1971, Monog. Nederl. Entom. Vereng., 5, pp.1-412. Hoffman, 1979, Mus. Hist. Nat. Genéve: 153, pp. 209; 1999, Virg. Mus. Nat. Hist. Spec. Public., 8: pp. 288. Pérez-Asso y Pérez-Gelabert, 2001, Bol. S.E.A., 28: 73-74. Pérez-Asso, 2005, Solenodon, 5: 53-59; 2009, Solenodon, 8: 33-81. PérezGelabert, 2008,ZOOTAXA 1831, 71, pp. 530.

\section{Achromoporus atrechoensis sp. nov.}

(Fig.1, A-G)

Diagnosis. Gonopodos con acropodito (fémur) birramoso, articulado en ángulo de $90^{\circ}$ con el prefémur y bifurcándose desde la región inferior (Fig.1, C-E). Sobrepasan ligeramente el borde anterior de S7. Las dos ramas del acropodito aproximadamente de la misma altura, la anterior (solenomerito) ancha en la base, corto extremo filiforme, doblado como "s" invertida; rama posterior laminar, con extremo suavemente espatulado (Fig.1, C y E). Proceso prefemoral complejo, más largo que el acropodito, expandido en porción media, con dos pequeños pliegues, uno anterior y otro posterior, la región superior es laminar con una concavidad mirando hacia región posterior del cuerpo, su extremo es asimétricamente redondeado con corto ápice agudo apuntando hacia la región posterior del cuerpo (Fig.1, C, D, F y G). Prefémur largo, región de articulación con el acropodito plegada (Fig.1, C-E). Cuerpo mediano (hasta $31.5 \mathrm{~mm}$ de longitud en hembras maduras), castaño rojizo, sin diferencia de color entre segmentos poríferos y no poríferos (Fig.1, A-B). Obvio dimorfismo sexual: región anterior de los machos (S2-S4) ligeramente más ancha que la posterior, mitad posterior de las hembras con segmentos convexos y metaterguitos levantados en el centro, dando aspecto globoso. Ausencia de setas en el dorso. Diente en AAP entre S2 y S7. Margen posterior de paraterguitos liso. Poros abriendo dorso-lateralmente desde un callo porífero no prominente en APP, fórmula porífera normal: 5,7,9-10,12-13,15-19. Paraterguitos amplios en machos y reducidos en hembras. 

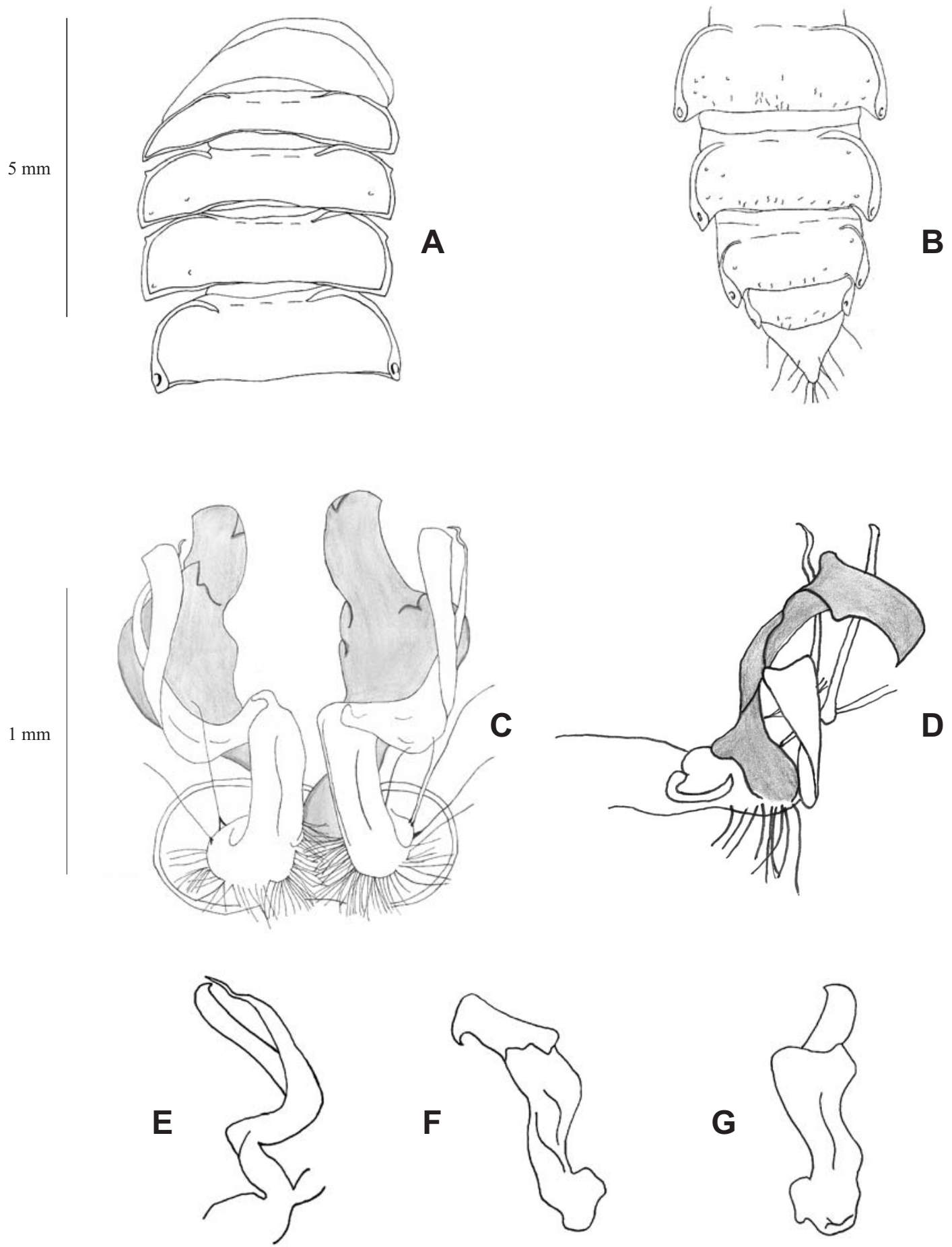

Figura 1. Cuerpo y gonopodos de Achromoporus atrechoensis sp. nov. A. Región anterior del cuerpo. B. Región posterior del cuerpo. C. Gonopodos, vista posterior. D. Gonopodo izquierdo, vista lateral anterior. E. Acropodito, vista anterior. F. Proceso prefemoral, vista anterior. G. Proceso prefemoral, vista lateral. A-C, holotipo (19.562); D-G, paratipo 19.484 . 
Diagnosis. Gonopods with acropodite bifurcated, union with prefemur forming a $90^{\circ}$ angle, the two branches separated from lower portion (Fig.1, C-E), slightly exceeding anterior margin of segment 7. Both acropodite branches similar in size, the anterior branch (solenomere) wide at the basi, apical portion short and filiform, like a "s" reversed; posterior branch laminated with apical portion slightly spatulated (Fig.1, C y E). Prefemoral process complex, longer than acropodite, wide at its central portion with two folds, anterior and posterior, superior portion laminated and concave, asymmetrically rounded with short and acute apex pointing towards posterior body region (Fig.1, C, D, F y G). Prefemur elongated, region of articulation with acropodite folded (Fig.1, C-E). Medium size (up to $31.5 \mathrm{~mm}$ in mature females), reddish brown, poriferous and non-poriferous segments with similar color pattern (Fig.1, A-B). Sexual dimorphism: anterior males' body (S2-S4) slightly wider than posterior region, posterior females' body convex, looking globose. Dorsum without setae. Anterior corner of paranota with tooth between segments 2 and 7, posterior margin smooth. Ozopore opening dorso-laterally from peritremata (callus,) non-prominent at posterior corner of paranota, pore formula normal (segments 5, 7, 9-10, 12-13, 15 to 19). Paranota wide in male, small in female.

Descripción del Holotipo. Macho (đ). Número catalográfico: MNHNSD19.562. Longitud=30.5 mm, anchura $=4.0 \mathrm{~mm}$. Figura 1,A-C.

Vista dorsal. Ligeramente más ancho en región anterior del cuerpo, color castaño rojizo uniforme. Ausencia de setas, algunos nódulos muy pequeños y velados dispersos en paraterguitos a partir de S3, algunos pliegues poco manifiestos en paraterguitos de segmentos posteriores. Paraterguitos amplios. APP inclinándose hacia atrás a partir de S11, los de S16-S19 son más largos. Poros abriendo dorso-lateralmente desde un callo porífero no prominente, con fórmula porífera normal: 5,7, 9-10, 12-13, 15-19. Diente pequeño en ángulo anterior o en margen lateral de paraterguitos en S3-S7, pero en S7 solo del lado derecho. Margen posterior de paraterguitos liso, sin nódulos ni dientes. Epiprocto castaño rojizo, con 6 pares de macrosetas erectas vistas desde arriba. Región superior de la cabeza negra con un par de macrosetas, una a cada lado de la sutura media. Antenas largas, alcanzando S4 cuando se extienden hacia atrás, castaño rojizas, pubescentes, último antenómero blanquecino.

Vista ventral. Epicráneo y frons castaño rojizo, clípeo y genas variando a un tono más claro; setas aisladas en frons y clípeo. Ancho de la cabeza, $3.7 \mathrm{~mm}$. Bases de las antenas con aproximadamente 1.2 $\mathrm{mm}$ de separación. Esternitos castaño-claro uniforme. Adaptación sexual secundaria: prominencia cónica sobre coxas del segundo par de patas, con pequeñas setas erectas en el extremo. Hypoprocto triangular con un par de macrosetas erectas, castaño claro, valvas anales ligeramente estriadas, castaño claro como hypoprocto, pigidio prominente, del mismo color que las valvas, con 2 pares de setas en el extremo. Gonopodos según la diagnosis.

Variabilidad $y$ regularidades morfológicas. Machos: longitud $=29.1 \mathrm{~mm} \quad(25.5-30.5 ; \mathrm{n}=6)$, anchura $=3.7 \mathrm{~mm}(3.2-4.0 ; \mathrm{n}=6)$. Hembras: longitud=29.0mm $(25.0-31.5 ; \mathrm{n}=4)$, anchura $=3.9(3.5-$ $4.1 ; \mathrm{n}=4)$.

Vista dorsal. La coloración solo varía de castaño rojizo a un tono más claro pero siempre uniforme, sin diferencias de color entre segmentos poríferos y no poríferos, en $\lesssim 484$ se observan manchas blancas en algunos segmentos y en 9563 una mancha negra en paraterguitos de $\mathrm{S} 2$ y S3, pero estos casos pudieran ser considerados como anormalidades. En todos los casos los machos son ligeramente más anchos en región anterior del cuerpo, las hembras presentan los segmentos posteriores convexos a partir de la mitad del cuerpo, tomando aspecto globoso. La cabeza y el collum son del mismo color que el resto del cuerpo o un poco más claros. Antenas pubescentes, castaño rojizas, último antenómero blanquecino, más largas en los machos, alcanzando S4 cuando se extienden hacia atrás, en las hembras no sobrepasan margen posterior de S3. Paraterguitos amplios y ligeramente levantados en los machos, reducidos y no levantados en las hembras, las cuales presentan los de $\mathrm{S} 14$ poco definidos ( $(+9563,1,025$ y 1,026). APP inclinándose hacia atrás a partir de S8, S9, S10 o S11 en machos, a partir de segmentos más posteriores en hembras, los de S16 en adelante más alargados. Nódulos muy pequeños, velados y dispersos en paraterguitos, no distinguibles en algunos segmentos y formando dos hileras paralelas en otros, tenues pliegues en algunos terguitos. Margen posterior de paraterguitos siempre liso. Diente muy pequeño en AAP o más abajo, en el margen lateral, 
S2-S5 (ðð561 y 1,126 y 우), S3- S7 (ðð562, 560), S3-S6 (ð559). El poro abre dorsolateralmente desde un callo porífero no prominente, callos más reducidos en hembras, fórmula porífera normal en todos los casos: 5, 7, 9-10,12-13,15-19. Epiprocto del mismo color que segmentos del cuerpo o un poco más claro, con 6 pares de macrosetas vistas desde arriba en todos los ejemplares, sin contar las del extremo del pigidio. Región superior de la cabeza con uno o dos pares de setas a ambos lados de la sutura media.

Vista ventral. Epicráneo, frons y clípeo castaño rojizo como el dorso y genas más claras, o el clípeo y las genas más claras; setas dispersas por toda esta región de la cabeza, siendo más largas en las hembras. Bases de las antenas apreciablemente separadas en machos y hembras. Adaptaciones sexuales secundarias invariables en los machos: prominencia cónica sobre coxas del segundo par de patas, con pequeñas setas en el extremo. El color de las patas varía de castaño a amarillentas, el tercer podómero más largo que los demás, más largo aún en las hembras. Color castaño claro, en las hembras los segmentos posteriores son casi blanquecinos. Hypoprocto sin variaciones, a no ser la tonalidad, de castaño claro a amarillo, siempre triangular con un par de macrosetas. Valvas anales siempre estriadas, con dos pares de macrosetas, uno de ellos junto al reborde de unión, su color puede variar de castaño a amarillento. Pigidio prominente, del mismo color que las valvas y el hypoprocto o más claro, con uno o dos pares de macrosetas en el extremo. Gonopodos sin variaciones obvias, que no sea la altura ligeramente mayor del proceso prefemoral en ${ }^{\lambda} 561$, en el que llega a alcanzar las patas posteriores de S6, en los demás ejemplares queda por debajo.

Etimología. El epíteto específico hace referencia a la loma El Atrecho, la que se levanta a pocos metros de la localidad tipo.

Comentarios. Achromoporus atrechoensis sp. nov. presenta dos de las características más destacadas en la descripción del sinonimizado género Lasiomazus Loomis, 1941, a saber, la articulación en $90^{\circ}$ entre el prefémur y el acropodito, y el desarrollo muy complejo del proceso prefemoral. Al asumir como correcta la reciente sugerencia de tomar el acropodito birramoso como criterio definitorio (Pérez-Asso, 2009), las referidas características pasan a constituir parte de la variabilidad dentro del género Achromoporus. Achromoporus atrechoensis sp. nov. presenta el mismo dimorfismo sexual de $A$. concolor (Loomis): dorso de las hembras ligeramente más convexo y paraterguitos de los machos más desarrollados; los ejemplares incluidos en esta descripción fueron colectados bajo gramíneas y arbustos en áreas de pinares en el Parque Nacional José del Carmen Ramírez, coincidiendo esto con el hábitat de los ejemplares de $A$. concolor colectados por Pérez-Asso y colaboradores en el Parque Nacional Valle Nuevo. Diplópodos asociados en las áreas de colecta: otros chelodésmidos, Stemmiulidae, Spirobolellidae y Rhinocricidae.

Tipos. Holotipo ${ }^{\Uparrow}$ MNHNSD 19.562 y paratipos $\widehat{\jmath} 19.484$, $q 19.563$, de la localidad tipo: mancha de bosque latifoliado entre pinares a pocos metros de la loma El Atrecho, en el camino de Alto de

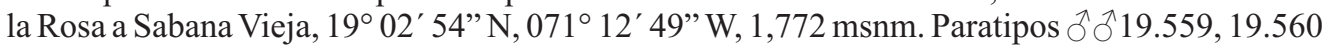
y 19.561, Alto de la Rosa, cerca de la Torre de Observación, $19^{\circ} 02^{\prime} 19^{\prime}$ N, $071^{\circ} 13^{\prime} 12^{\prime}$ 'W, 1,792 msnm. Paratipo ${ }^{\Uparrow}$ juvenil 19.1,126, cerca de Piedra del Aguacate, desde Alto de la Rosa, en cañada de bosque latifoliado entre pinares, $19^{\circ} 02^{\prime} 19.4^{\prime \prime} \mathrm{N}, 071^{\circ} 11^{\prime} 39.7^{\prime}$ ' $\mathrm{W}, 1,633 \mathrm{msnm}$. Paratipos 9 ㅇ 19.1,025, 19.1,026 y 19.1,031 (juvenil), en pinar cerca de la Torre de Observación, en el camino de Alto de la Rosa a Sabana Vieja, 19 $02^{\prime} 21^{\prime \prime}$ N, 071 ${ }^{\circ} 13^{\prime} 20.8^{\prime \prime}$ W, 1,792 msnm. Todas las localidades están dentro de los límites del Parque Nacional José del Carmen Ramírez, en la Cordillera Central de la República Dominicana. Colectores: Gabriel de los Santos, Elvi de los Santos, Miguel A. Landestoy, Robert Ortiz y Carlos Suriel, durante dos expediciones: (2629)-vi-08 y (21-25)-xi-09. Los especímenes fueron depositados en la colección de diplópodos del Museo Nacional de Historia Natural, Santo Domingo (MNHNSD), excepto los paratipos đ19.560 y q 19.1,025 donados en intercambio a la colección de diplópodos ARPA.

Distribución geográfica. Solo conocida de las localidades referidas para el holotipo y los paratipos. 


\section{Achromoporus elegans sp. nov.}

(Fig. 2, A-G)

Diagnosis. Gonopodos gruesos, relativamente cortos en relación con su grosor, pero sobrepasando ampliamente el borde anterior de S7. Acropodito (fémur) birramoso, con la bifurcación en región subterminal (Fig. 2, C-E). Rama anterior del acropodito (solenomerito) ligeramente más larga que la rama posterior y sobrepasando mínimamente la región más elevada del proceso prefemoral, arqueada, extremo semitruncado con ángulo interior apiculado y ángulo exterior redondeado, canal prostático visible en cara interna. Rama posterior lanceolada y arqueada, extremo muy agudo (Fig. 2, C y E). Proceso prefemoral con forma de copa en la porción inferior, forma de " $u$ " en la porción media, con prominente pliegue ventral, porción distal alargada, torcida, con extremo ampliamente espatulado (Fig. 2, C, D, F y G). Prefémur corto, plegado hacia dentro (Fig. 2, C y E). Cuerpo grande, ligeramente más ancho en región media y posterior, hembras alcanzando hasta $44 \mathrm{~mm}$ de longitud y machos hasta $37 \mathrm{~mm}$. Dorso sin setas. Terguitos convexos, con nódulos casi imperceptibles, formando dos muy veladas hileras paralelas. Proterguitos, metaterguitos y pleuritos básicamente negros. Segmentos poríferos y no poríferos diferentes, paraterguitos blancos en segmentos poríferos, negros con solo el margen lateral blanco en segmentos no poríferos; collum con los ángulos blancos. Ausencia de dientes en paraterguitos. Margen posterior de paraterguitos liso (Fig. 2, A-B). Poro abriendo lateralmente desde un callo porífero poco prominente, fórmula porífera normal: 5,7, 9-10, 12 13, 15-19. APP básicamente rectos. Epiprocto negro o castaño rojizo en porción anterior, más claro en el extremo. Cabeza negra o castaño en región superior. Patas rojizas y pubescentes, con setas cortas y erectas, el tercer podómero en los machos tiene engrosamiento en región basal y ventral. Machos con adaptación sexual secundaria en primer par de patas, prominencia o proceso cónico pronunciado en coxas del segundo par. Hypoprocto redondeado, hinchado, con un par de macrosetas, valvas anales estriadas.

Diagnosis. Gonopods thick, short in relation to thickness, but exceeding anterior margin of segement 7 enough. Acropodite bifurcated, the two branches separated at subapical portion (Fig. 2, C-E). Anterior branch (solenomere) longer than posterior branch and exceeding slightly the higher region of prefemoral process, curved, truncated apically with inner angle acute and external angle rounded, obvious prostatic groove on inner side. Posterior branch lanceolated and curved, apical portion acute (Fig. 2, C y E). Prefemoral process cup-shaped at lower portion, "u" shape at its central portion, with prominent ventral fold, upper portion elongated, twisted, largely spatulated apically (Fig. 2, C, D, F y G). Prefemur short and folded inwards (Fig. 2, C y E). Large size, lightly wider at the middle and posterior region than the anterior body, up to $44 \mathrm{~mm}$ in females and $37 \mathrm{~mm}$ in males. Dorsum without setae. Body convex, with very small nodules, nearly imperceptible, arranged in two parallel rows. Proterga, metaterga and pleurites black. Poriferous and non-poriferous segments different, paranota white in poriferous segments and black with only lateral margin white in non-poriferous segments; collum with white angles. Paranota dentation absent. Posterior margin of paranota smooth and glabrous (Fig. 2,A-B). Ozopore opening laterally from peritremata (callus,) non-prominent at posterior corner of paranota, pore formula normal (segments 5, 7, 9-10, 12-13, 15 to 19). Epiproct black or reddish brown at its anterior part, light brown backwards, with long setae apically. Head black or chestnut at the upper area. Legs reddish with short and erect setae, third males' podomere with basal and ventral thickening. Males'coxae of the second pair of legs with conical process. Hypoproct rounded, swollen, with two long setae, anal valves striated.

Descripción del Holotipo. Macho (đ̋). Número catalográfico MNHNSD 19.485. Longitud=37.0 mm, anchura $=5.5 \mathrm{~mm}$. Figura 2, A-C.

Vista dorsal. Cabeza ancha, $3.8 \mathrm{~mm}$, negra en región superior, epicráneo y frons, genas más claras, clípeo castaño. Dos pares de setas a ambos lados de la sutura media en la región superior de la cabeza, otros dos pares de setas a nivel del frons, a ambos lados de la sutura. Setas más pequeñas dispersas por todo el frons y el clípeo. Antenas gruesas, castaño rojizas, pubescentes, alcanzando S4 cuando se extienden hacia atrás, separadas por $0.8 \mathrm{~mm}$. Setas largas en primer antenómero. Terguitos convexos, sin setas, metaterguitos negros, paraterguitos blancos en segmentos poríferos y negros con solo el borde blanco en los no poríferos, collum con ángulos blancos. Metaterguito de S7 más claro. Nódulos poco definidos, forman una hilera en el collum, paralelamente al margen anterior, a partir de S8 se definen veladamente en dos hileras paralelas. Poro abriendo lateralmente desde callo no prominente en APP, fórmula porífera normal, 5,7, 9-10, -12-13, 15-19. APP recto hasta S15, muy pequeño en S19, casi oculto por paraterguito S18. Ausencia de dientes. Prozonitos y pleuritos negros. Epiprocto negro, con seis pares de setas vistas desde arriba. Pigidio prominente, con dos pares de macrosetas en su extremo. 


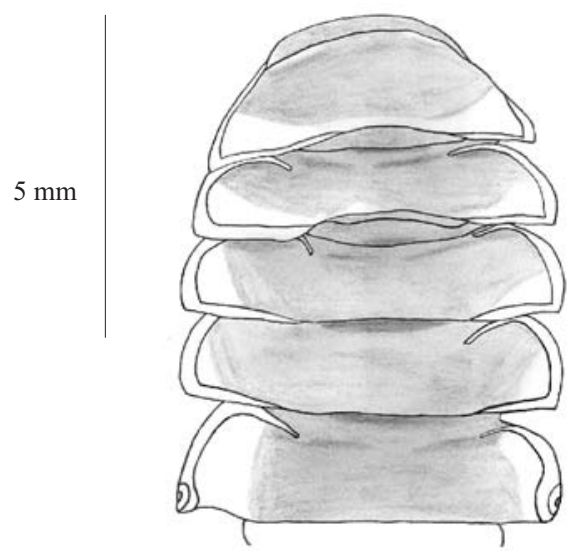

A

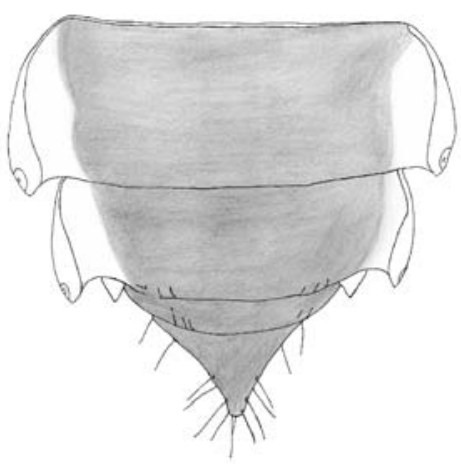

B
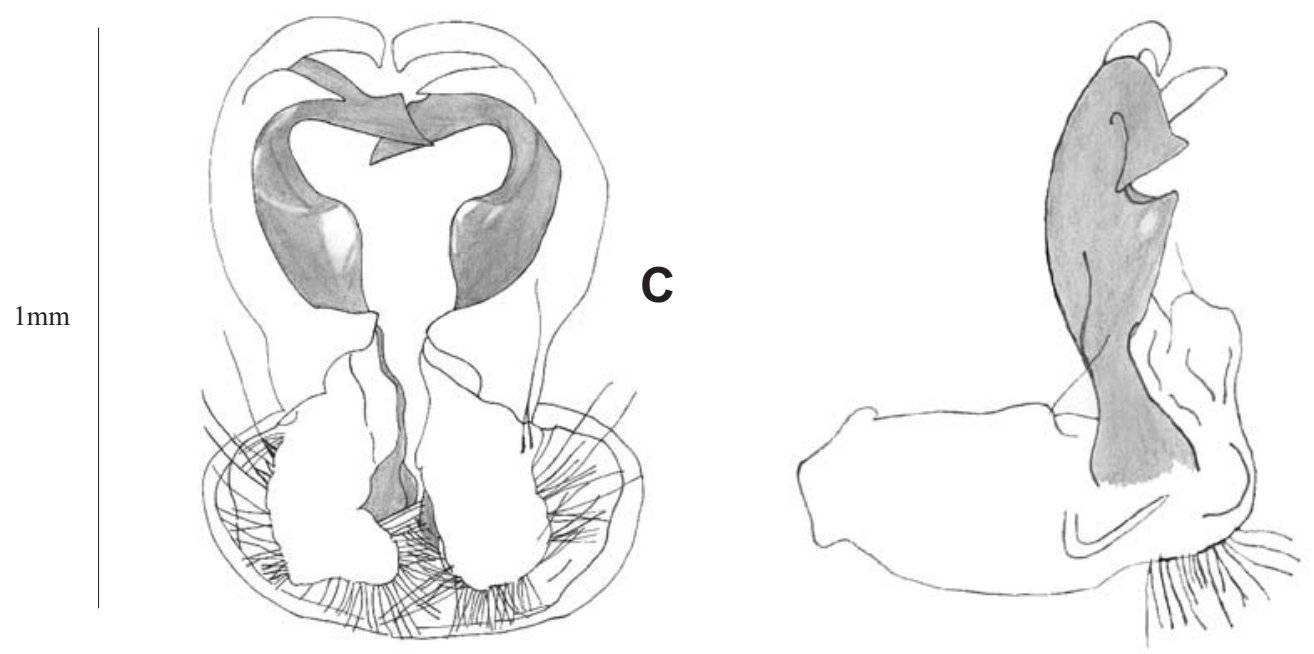

D
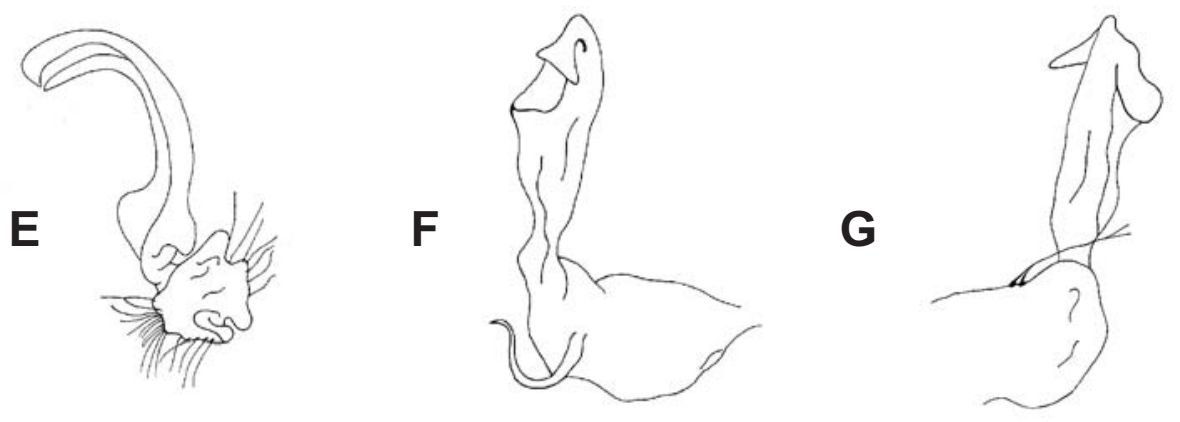

Figura 2. Cuerpo y gonopodos de Achromoporus elegans sp. nov. A. Región anterior del cuerpo. B. Región posterior del cuerpo. C. Gonopodos, vista posterior. D. Gonopodo izquierdo, vista lateral anterior. E. Acropodito, vista anterior. F. Proceso prefemoral, vista lateral-posterior. G. Proceso prefemoral, vista lateral anterior. A-C, holotipo (19.485); D-G, paratipo 19.964 . 
Vista ventral. Esternitos castaño rojizo, con setas en porción anterior y glabros en porción posterior. Patas gruesas y largas, castaño rojizas. Tercer podómero presentando expansión lateral en su porción basal y ventral, con pequeño callo. Protuberancia cónica en coxas de segundo par de patas. Macroseta erecta en cara ventral del tercer podómero a partir del tercer par de patas. Hypoprocto redondeado y grueso, castaño claro, con un par de setas erectas. Valvas anales negras y estriadas, con dos pares de setas, uno sobre el reborde de unión y otro a cada lado de éste. Gonopodos según la diagnosis.

Variabilidad y regularidades morfológicas. Machos: longitud= $33.57(31-37 ; \mathrm{n}=7)$, anchura=4.44 (4.0$5.5 ; \mathrm{n}=7)$. Hembras: longitud=40.43 (38-44;n=7), anchura=6.78 (6.0-7.5;n=7).

Vista dorsal. Cabeza variando entre negro y castaño oscuro en región superior, con dos pares de setas en ambos lados de la sutura media. Collum y metaterguitos negros o castaño rojizo, los segmentos poríferos S5, S7, S9 y S10 son de tono más claro, los segmentos posteriores suelen ser más oscuros. La diferencia de color en segmentos poríferos y no poríferos se mantiene regular según diagnosis. Terguitos apreciablemente convexos, más anchos en los segmentos posteriores, principalmente en las hembras; en خ965 los segmentos posteriores son ligeramente más estrechos, probablemente por tratarse de un macho juvenil. Prozonitos y pleuritos variando de negro a castaño rojizo. Presencia de nódulos poco definidos a partir del collum, muy velados en algunos especímenes, cuando aparecen definidos forman una hilera paralela al borde anterior del collum o del metaterguito, en los segmentos posteriores suelen apreciarse formando dos hileras paralelas, algunos dispersos en paraterguitos; el collum de $\$ 1,038$ presenta dos hileras semiparalelas definidas. No hay setas en ninguno de los terguitos. Los poros abren lateralmente desde un callo no prominente, en un caso no se aprecian fácilmente en vista dorsal desde arriba ( 91,040$)$. Fórmula porífera normal sin variación. APP recto en segmentos anteriores, el de S19 muy reducido y medio oculto por el de S18. Ausencia de dientes en AAP en todos los casos. Terguitos gruesos. Borde posterior de metaterguitos a veces con tenues pliegues en segmentos posteriores, originados por los

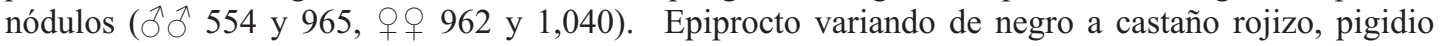
pronunciado, siempre más claro.

Vista ventral. Frons negro o castaño rojizo, clípeo y genas más claras, setas dispersas en toda esta región, a veces profusas en el labrum. Antenas más largas en machos, en los que sobrepasa S3 cuando se extienden hacia atrás, en las hembras no sobrepasa el borde posterior de S3. Adaptación sexual secundaria sobre coxa del segundo par de patas en todos los machos examinados. Macroseta sobre el trocánter a partir del cuarto par de patas en ambos sexos, ligeramente inclinada hacia atrás. Las patas son rojizas, básicamente en los últimos podómeros. Tanto machos como hembras presentan una hilera de setas cortas y erectas en esternitos, a nivel del primer par de patas del segmento, reduciéndose en segmentos posteriores. Hypoprocto castaño claro, redondeado, engrosado y con un par de setas largas y erectas, más engrosado en las hembras, en $\widehat{\nearrow} 965$ aparece muy atrofiado, casi inexistente. Valvas anales varían de negro a castaño rojizo y amarillento, estriadas y con dos pares de setas, un par sobre la sutura de unión y otro par a cada lado de esta. Pigidio pronunciado, siempre más claro, castaño rojizo o amarillo, más frecuentemente con dos pares de setas en su extremo; + 1,038 presenta tres pares de setas. Los gonopodos no presentan variaciones, a no ser la tonalidad de su color amarillo una vez preservados en alcohol, y el extremo expandido del proceso prefemoral, el que aparece muy hendido, casi bifurcado, en $\precsim 1,115$.

Etimología. El epíteto específico hace referencia a la belleza de los ejemplares de esta especie.

Comentarios. Achromoporus elegans sp. nov. es el segundo más grande de los chelodésmidos descritos para La Hispaniola, siguiendo a A. magnus Pérez-Asso, 2009. Excepto uno, todos los ejemplares colectados estaban enterrados entre raíces de árboles o dentro de troncos en descomposición. Diplópodos asociados en el área de colecta: Stemmiulidae y Pyrgodesmidae.

Tipos. Holotipo: ${ }^{\lambda}$ MNHNSD 19.485 mancha de bosque latifoliado entre pinares, camino de Alto de la Rosa a Sabana Vieja, cerca de loma El Atrecho, $19^{\circ} 02^{\prime} 54^{\prime \prime} \mathrm{N}, 071^{\circ} 12^{\prime} 49^{\prime \prime} \mathrm{W}, 1,772 \mathrm{msnm}$ (localidad tipo). Paratipos ô $\partial^{\lambda} 19.554,19.1,039$ y $q$ q $19.555,19.1,040$, mancha de bosque latifoliado entre pinares, camino de Alto de la Rosa a Sabana Vieja, cerca de la Torre de Observación, 19 02' 19" N, 071 '13' 12" W, 1,792 msnm. Paratipos $\circ$ $\uparrow 19.1,037$ y 19.1,038, cerca de la Torre de Observación, en la misma área de

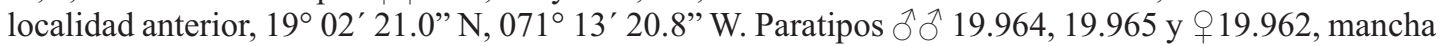
de bosque latifoliado entre pinares en camino de Alto de la Rosa a Sabana Vieja, un poco antes de la 


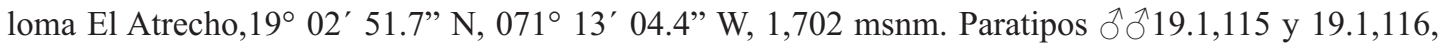
우19.1,117 y 19.1,118, cerca de Piedra del Aguacate, desde Alto de la Rosa, en cañada de bosque latifoliado entre pinares, $19^{\circ} 02^{\prime} 19.4^{\prime \prime} \mathrm{N}, 071^{\circ} 11^{\prime} 39.7^{\prime \prime} \mathrm{W}, 1,633 \mathrm{msnm}$. Todas las localidades referidas se encuentran dentro del Parque Nacional José del Carmen Ramírez. Las colectas se realizaron durante dos expediciones: (26-29)-vi-08 y (21-25)-xi-09. Colectores: Gabriel de los Santos, Elvi de los Santos, Miguel A. Landestoy, Robert Ortiz y Carlos Suriel. Los especímenes tipo se depositaron en la colección de diplópodos del Museo Nacional de Historia Natural, excepto los paratipos ふ̋19.1,116 y † 19.1,118, cedidos en intercambio a la colección ARPA.

Distribución. Achromoporus elegans sp.nov solo se ha colectado en las localidades referidas para los tipos.

\section{AGRADECIMIENTOS}

Gabriel de los Santos tomó las fotografías al microscopio que sirvieron al autor para los dibujos del dorso y gonopodos completos, además participó en las colectas y ha sido un buen colaborador en los trabajos de campo. Antonio R. Pérez-Asso revisó el manuscrito y examinó los especímenes haciendo importantes sugerencias. Celeste Mir auspició las expediciones e hizo correcciones al texto en inglés del Abstract y las Diagnosis. Altagracia Espinosa revisó el manuscrito. Brian Farrell (MCZ, Harvard University) y Manuel Valdez (Instituto de Investigaciones Botánicas y Zoológicas, Universidad Autónoma de Santo Domingo) facilitaron los equipos fotográficos y de microscopía, así como los programas de procesamiento para las fotos. El Viceministerio de Áreas Protegidas y Biodiversidad expidió los permisos de colecta, con las mediaciones de Aleyda Capella. Robert Ortiz, Miguel A. Landestoy y Elvi de los Santos ayudaron en las colectas. Rafael y Orlando Sánchez sirvieron de guías en las montañas. De todos ellos nos sentimos agradecidos.

\section{LITERATURA CITADA}

Hoffman, R. L. 1979. Clasification of the Diplopoda. Mem. Mus. His. Nat. Geneve. 209 pp.

Hoffman, R. L. 1999. Checklist of the millipedes of North and Middle America. Virg. Mus. Nat. Hist. Spec. Public., 8: 288.

Jeekel, C. A. W. 1971. Nomenclator generum et familiarum Diplopodorum: A list of the genus and familygroup names in the Class Diplopoda from the $10^{\text {th }}$ edition of Linnaeus 1758, to the end of 1957. Nederl. Ent. Ver., Amsterdam, 5: 267 En http://www.biologie.uni-ulm.de/systax/,

Loomis, H. F. 1936. The millipeds of Hispaniola, with descriptions of a new family, new genera, and new species. Bull. Mus. Comp. Zool., 80 (1): 3-197.

Loomis, H. F. 1941. Millipeds collected in Puerto Rico and the Dominican Republic by Dr. P. J. Darlington in 1938. Bull. Mus. Comp. Zool., 88 (2): 17-80.

Pérez-Asso, A. R. 1996. Revisión del género Amphelictogon (Diplopoda: Polydesmida: Chelodesmidae) en Cuba. Insecta Mundi, 10:(1-4), 181-216.

Pérez-Asso, A. R. 2005. Dos especies y una subespecie nueva de milpiés del género Achromoporus (Diplopoda: Polydesmida) para Hispaniola. Solenodon, 5: pp. 53-59.

Pérez-Asso, A. R. 2009. El género Achromoporus (Diplopoda: Polydesmida: Chelodesmidae) en República Dominicana: especies nuevas y sinonimias. Solenodon, 8: 33-81.

Pérez-Asso, A. R. y D. E. Pérez-Gelabert 2001. Checklist of the millipeds (Diplopoda) of Hispaniola. Boletín S.E.A. 28: 67-80.

Pérez-Gelabert, D. E. 2008. Arthropods of Hispaniola (Dominican Republic and Haiti): A checklist and bibliography. ZOOTAXA 1831, 71, pp. 530. 